\title{
Ultrasound-guided single erector spinae plane block versus thoracic paravertebral block for patients undergoing video-assisted thoracoscopic lobectomy: a randomized controlled trial
}

\section{Danxu Ma}

Beijing Chaoyang Hospital

\section{Huilong Ren}

Beijing University of Chinese Medicine Affiliated Dongzhimen Hospital

\section{Xueyang Li}

Beijing Chaoyang Hospital

Huili Li

Beijing Chaoyang Hospital

Jia Jiang

Beijing Chaoyang Hospital

Anshi Wu

Beijing Chaoyang Hospital

Yun Wang ( $\square$ wangyun129@ccmu.edu.cn )

Beijing Chaoyang Hospital https://orcid.org/0000-0003-0695-8861

\section{Research article}

Keywords: Ultrasound-guided, Erector Spinae Plane Block, Thoracic Paravertebral Block, video-assisted thoracoscopic lobectomy, Patient-controlled intravenous analgesia

Posted Date: September 26th, 2019

DOI: https://doi.org/10.21203/rs.2.14933/v1

License: (c) (1) This work is licensed under a Creative Commons Attribution 4.0 International License. Read Full License 


\section{Abstract}

Background: Whether erector spinae plane (ESP) block can replace thoracic paravertebral block (TPVB) remains unknown. This study aimed to determine the efficacy and safety of ultrasound-guided ESP block compared with TPVB for postoperative analgesia after video-assisted thoracoscopic lobectomy under general anesthesia.

Methods: This prospective randomized controlled trial divided patients into a control group, a TPVB group ( $0.3 \mathrm{~mL} / \mathrm{kg}, 0.5 \%$ ropivacaine), and an ESP group ( $0.5 \mathrm{~mL} / \mathrm{kg}, 0.5 \%$ ropivacaine). Dermatomes with loss of pinprick sensation, heart rate, and mean arterial pressure were recorded during 30 min after block administration. Visual analog scale (VAS) scores, total analgesic dose, and complications after surgery were recorded.

Results: No obvious hemodynamic fluctuations occurred in the TPVB and ESP groups within 30 min after block administration. The segmental dermatomes with loss of pinprick sensation $30 \mathrm{~min}$ after regional block were 4.5 (1.1) dermatomes (T3-T7) in the TPVB group and 5.4 (1.2) dermatomes (T2-T8) in the ESP group. At 1 and $6 \mathrm{~h}$ after operation, VAS scores were lower in the TPVB group than in the control group (rest: $1 h, P<0.001,6 h, P=0.004$; cough: $1 h, P<0.001,6 h, P<0.001$ ), but at 18,24 , and $48 h$, there was no significant difference (rest: 18h, $P=0.18,24 h, P>0.99,48 h, P>0.99$; cough: $8 h, P>0.99,24 h, P>0.99$, 48h, $P>0.99$ ). VAS scores were similar in the TPVB and ESP groups at $1 \mathrm{~h}$ postoperatively (rest: $\mathrm{P}>0.99$, cough: $P>0.99$ ), but were lower in the ESP group at $6,18,24$, and $48 \mathrm{~h}$ (rest: $6 \mathrm{~h}, \mathrm{P}=0.018,18 \mathrm{~h}, \mathrm{P}<0.001,24 \mathrm{~h}, \mathrm{P}<$ 0.001 , 48h, $P<0.001$; cough: 6h, $P=0.004$, 18h, $P<0.001$, 24h, $P=0.004,48 h, P=0.003$ ). The doses of postoperative patient-controlled analgesia and rescue analgesia were lower in the TPVB and ESP groups than in the control group and were lower in the ESP group than in the TPVB group No block-related complications occurred.

Conclusions: Single ESP block provided superior postoperative analgesia than TPVB, without causing any adverse effect.

Trial registration: The study was approved by the ethics committee of Beijing Chaoyang Hospital (2017ke-81). The trial was registered retrospectively at the Clinical Trial Registry of China (ChiCTR-INR17011499) on May 26, 2017.

\section{Background}

Thoracic surgery is considered to be one of the most painful surgical procedures. Pain after thoracic surgery can lead to delayed recovery and increase the incidence of complications such as atelectasis and pulmonary infections due to ineffective breathing and clearing of secretions. Effective pain management may reduce these complications and the risk of chronic pain[1-3].

Thoracic epidural analgesia and thoracic paravertebral block (TPVB) are widely used for postoperative analgesia after thoracotomy. However, these procedures are technically challenging and associated with 
high failure rates[4]. Thoracic epidural analgesia is associated with complications such as spinal cord trauma, epidural hematoma, hypotension, and respiratory depression[5], while TPVB is associated with a risk of pneumothorax[6].

Erector spinae plane (ESP) block was first reported by Forero et al. in 2016[7]. ESP block may be safer than paravertebral block, because the injection is in a plane that is far from critical structures such as the pleura[8]. In clinical studies, ESP blocks have produced extensive cutaneous sensory block, indicating that both the ventral and dorsal spinal rami were anesthetized $[9,10]$. Furthermore, a cadaver study showed that a dye injected into the ESP spread up to the origins of the ventral and dorsal rami of the spinal nerves[7]. The local anesthetics injected for ESP blocks may also reach and block the rami communicantes and sympathetic chain to produce visceral analgesia[11-13]. This raises the question of whether the ESP block can serve as a technically simpler alternative to TPVB with a similar analgesic effect.

The aim of this study was to determine the safety and efficacy of ESP block in comparison to the TPVB for relieving acute pain in patients undergoing video-assisted thoracoscopic lobectomy.

\section{Methods}

Patients and study design

This prospective randomized controlled study was approved by the ethics committee of Beijing Chaoyang Hospital (2017-ke-81) and registered with the Clinical Trial Registry of China (ChiCTR-INR17011499). The study took place at the Department of Anesthesiology, Beijing Chaoyang Hospital, Capital Medical University, Beijing, from Jun 1, 2017, to May 30, 2018. All patients provided written informed consent before being enrolled into the study.

Patients were eligible for enrollment in this study if they were between 20 and 70 years of age, were scheduled to undergo video-assisted thoracoscopic lobectomy, and had an American Society of Anesthesiologists (ASA) class of I or II. Patients with local infection, coagulopathy, allergy to local anesthetics, morbid obesity (body mass index $>30 \mathrm{~kg} / \mathrm{m}^{2}$ ), decreased pulmonary reserve, major cardiac disorders, renal dysfunction, pre-existing neurological deficits, or psychiatric illness were excluded. Patients who experienced severe intra- or postoperative bleeding or who required postoperative mechanical ventilation were also excluded from the study.

Randomization and blinding

The patients were randomized using sequential sealed envelopes prepared by an independent statistician using a computer-generated random number table. Patients were randomly allocated to an ESP group, a TPVB group, and a control group using computer-generated random numbers. Patients in the control group did not receive any regional block. Patients in the TPVB group received TPVB with $0.3 \mathrm{~mL} / \mathrm{kg}$ of 
$0.5 \%$ ropivacaine, while patients in the ESP group received ESP block with $0.5 \mathrm{~mL} / \mathrm{kg}$ of $0.5 \%$ ropivacaine. The postoperative assessors were blinded to grouping.

\section{Procedures}

The ESP and TPVB blocks were performed under ultrasound guidance with a Navi Series ultrasound device (Wisonic Medical Technology Co. Ltd., Shenzhen, China) equipped with a linear ultrasound transducer (4-15 MHz). Starting from 30 min after block administration, pinprick sensation in the T1T12 dermatomal distribution was monitored every $5 \mathrm{~min}$ by an independent observer who was blinded to the study group. After confirming sensory loss, general anesthesia was induced with midazolam 0.02 $\mathrm{mg} / \mathrm{kg}$, sufentanyl $0.3-0.4 \mu \mathrm{g} / \mathrm{kg}$, and propofol $1.5-2 \mathrm{mg} / \mathrm{kg}$. Rocuronium bromide $0.8 \mathrm{mg} / \mathrm{kg}$ was administered to facilitate tracheal intubation. Anesthesia was maintained with propofol and remifentanil, and the dosage was adjusted according to the hemodynamic parameters and bispectral index. Additional boluses of intravenous rocuronium bromide were given as required. During the 10 min before the end of the operation, only tramadol $100 \mathrm{mg}$ was used for analgesia. Non-steroidal anti-inflammatory drugs were not used during the operation.

Postoperative analgesia via a patient-controlled intravenous analgesia (PCIA) device was initiated immediately after the surgery and continued until $48 \mathrm{~h}$ after the surgery. The PCIA device contained 100 $\mu \mathrm{g}$ sufentanyl and $10 \mathrm{mg}$ tropisetron diluted with normal saline to a volume of $100 \mathrm{~mL}$. The device was set up to administer a background dose of $1 \mathrm{~mL}$ and a bolus dose of $2 \mathrm{~mL}$ with a 15-min lockout interval. Before the operation, patients were taught how to use the PCIA device to manage their pain and the visual analog scale (VAS) to score their pain. If the PCIA device could not meet a patient's analgesic demand, and the VAS score at rest was 4 or more, $50 \mathrm{mg}$ flurbiprofen axetil was intravenously administered as rescue analgesia.

Heart rate and mean arterial pressure were measured before block administration and 5, 15, and 30 min after block administration. An investigator blinded to the group allocation recorded the VAS scores at rest and during coughing at $1,6,18,24$, and $48 \mathrm{~h}$ after the operation. The number of doses administered via the PCIA device, the total volume of analgesic drugs administered via the PCIA device, the volume of flurbiprofen axetil administered, and the incidence of postoperative nausea and vomiting during the first $24 \mathrm{~h}$ and $48 \mathrm{~h}$ after the surgery were noted.

Statistical analysis

Statistical analysis was performed using IBM SPSS Statistics, version 22 (IBM Corp, Armonk, NY). Numerical data were expressed as mean and standard deviation or median and range, as appropriate. Qualitative data were expressed as frequency and percentage. Qualitative parameters were analyzed using the chi-square test or Fisher exact test. Quantitative parameters were compared between groups by using the independent-samples t-test or Mann-Whitney U-test. Each group was tested separately for repeated measures, which were evaluated using analysis of variance for repeated measures or the 
Friedman test, followed by a suitable post-hoc test. All tests were two-tailed. $\mathrm{P}<0.05$ was considered statistically significant.

\section{Results}

General information

A total of 81 patients were possibly eligible for enrollment into the study. Of these, 3 patients did not meet the selection criteria, and 11 patients did not consent to participate in the study. The remaining 67 patients were enrolled in the present study. All of them were scheduled to undergo video-assisted thoracoscopic lobectomy under general anesthesia and were randomly allocated to the three study groups. The block could not be administered in two patients. Additionally, four patients required conversion to open thoracic surgery, and one patient developed postoperative hemorrhage. Therefore, 60 patients were included in our final analysis. Of these, 19 patients were randomized to the control group, 20 patients were randomized to the TPVB group, and 21 patients were randomized to the ESP group. A flowchart of patient selection and the study procedures has been presented in Figure 1. Patients in each of the three groups were comparable with regards to age, sex, body mass index, ASA status, and duration of surgery (Table 1$)$.

\section{Outcomes}

Within 30 min after block administration, no significant fluctuations in hemodynamic parameters were observed in the TPVB or ESP group (Table 2). At 30 min after block administration, pinprick sensation was absent in 5.4 (1.2) dermatomes (T2-T8) in the ESP group and 4.5 (1.1) dermatomes (T3-T7) in the TPVB group.

At 1 and $6 \mathrm{~h}$ after the surgery, the VAS scores at rest and during coughing were significantly lower in the TPVB group than in the control group (rest: $1 \mathrm{~h}, P<0.001,6 \mathrm{~h}, P=0.004$; cough: $1 \mathrm{~h}, P<0.001,6 \mathrm{~h}, P<0.001$ ); however, no significant differences were seen between the two groups at 18,24 , and $48 \mathrm{~h}$ after the surgery (rest: $18 \mathrm{~h}, P=0.18,24 \mathrm{~h}, P>0.99,48 \mathrm{~h}, P>0.99$; cough: 8h, $P>0.99,24 \mathrm{~h}, P>0.99$, 48h, $P>0.99$ ). The VAS scores at rest and during coughing did not differ between the TPVB and ESP groups at $1 \mathrm{~h}$ postoperatively (rest: $P>0.99$, cough: $P>0.99$ ); however, at $6,18,24$, and $48 \mathrm{~h}$, these scores were significantly lower in the ESP group than in the TPVB group (rest: $6 \mathrm{~h}, P=0.018,18 \mathrm{~h}, P<0.001,24 \mathrm{~h}$, $P<0.001$, 48h, $P<0.001$; cough: $6 \mathrm{~h}, \mathrm{P}=0.004,18 \mathrm{~h}, P<0.001,24 \mathrm{~h}, P=0.004,48 \mathrm{~h}, P=0.003$ ) (Fig. 2 and 3 ).

The number of PCIA doses delivered, the volume of analgesic drugs administered via the PCIA device were significantly lower in the TPVB and ESP groups than in the control group $(P<0.001)$, and were lower in the ESP group than in the TPVB group ( $P<0.001)$ ( Fig. 4 and 5). The consumption of flurbiprofen axetil were significantly lower in the TPVB and ESP groups than in the control group (0-48h: TPVB vs Control, $P$ $=0.026$; ESP vs Control, $P<0.001)$, and were lower in the ESP group than in the TPVB group $(0-48 \mathrm{~h}: P=$ 0.028) (Fig. 6). Nausea and vomiting were the only postoperative complications in our patient cohort (Table 3). No block-related complications occurred. 


\section{Discussion}

This prospective randomized study showed that a single ESP block provided superior postoperative analgesia than the TPVB in patients undergoing video-assisted thoracoscopic lobectomy, without causing any adverse effects. To our knowledge, this is the first randomized controlled trial to compare the effects of ESP block and TPVB.

In our study, postoperative pain control was superior in the ESP group as compared to the TPVB group during 6-48 $\mathrm{h}$ after the operation, but not at $1 \mathrm{~h}$. This implies that the effects of the ESP block were more persistent than those of the TPVB. This may occur because an anesthetic drug injected into the space between the transverse process and the erector spinae muscle may only gradually penetrate anteriorly through the intertransverse connective tissues and into the paravertebral space[11, 12].

Interestingly, the slower effect was observed despite the higher anesthetic dose in the ESP group than in the TPVB group. We had performed a preliminary experiment prior to this study which showed that ESP block with $0.3 \mathrm{~mL} / \mathrm{kg}$ ropivacaine resulted in a narrow distribution of sensory loss (tested using pinprick sensation) at $20 \mathrm{~min}$ after administration. When the block was performed with a dose of $0.5 \mathrm{~mL} / \mathrm{kg}$ ropivacaine, obvious loss of pinprick sensation over a wide area could be measured within $20 \mathrm{~min}$. The optimal analgesic effect of TPVB can be achieved after a single ropivacaine dose of $0.3 \mathrm{~mL} / \mathrm{kg}$, according to previous studies[14, 15].

There is some debate about the mechanism of action of ESP block in the literature. Cadaveric and clinical evidence suggests that the local anesthetic may penetrate anteriorly through the intertransverse connective tissues into the paravertebral space where it blocks the ventral rami of the spinal nerves. Additionally, the anesthetic may reach and block the rami communicantes and sympathetic chain to produce visceral analgesia[11, 12]. However, Ivanusic et al. observed the dye-distribution pattern after ESP administration in 10 cadavers, and they found that dye couldn't spread to the anterior of the paravertebral space to involve the origins of the ventral and dorsal branches of the thoracic spinal nerves[16]. Adhikary et al. studied the distribution pattern after ESP administration in three fresh cadavers, and they concluded that the clinical effects of ESP block can be explained by the epidural and neural foraminal spread of the local anesthetic[17]. Of course, a cadaveric model cannot accurately reflect the spread of local anesthetic in a living body. The mechanism of ESP block may be clarified in future studies, but it would seem that it is not possible to comment on this aspect in the present study[18-20].

There were no block-related complications in our study. There was no significant hypotension in ESP group and TPVB group after 30 minutes of blockade, which were superior to epidural block as we know. Indeed, the only complications observed were nausea and vomiting. This suggests that the ESP block is safe for use in patients undergoing video-assisted thoracoscopic lobectomy. Additionally, the ESP block is less technically challenging than the TPVB. The erector spinae muscle can be very easily imaged using a low-frequency curved-array transducer. There are no important vessels, nerves, or organs over the transverse process. This can potentially reduce the risk of complications such as hematoma, nerve injury, 
pneumothorax, and block failure[21]. Further studies are needed to evaluate the efficacy of ESP block as a sole anesthetic technique.

There are several limitations to the current study. First, due to safety and time limitations, we only evaluated the block range 30 min after the block, but whether the block plane had been stabilized at this time needs further study. Second, we did not perform catheter insertion to prolong the analgesic effect of the local anesthetic. This is because the study was designed to assess the effects of a single injection. We will evaluate continuous ESP block in future studies.

\section{Conclusions}

ESP block appeared to be a good alternative for postoperative pain management in patients undergoing video-assisted thoracoscopic lobectomy. Compared to TPVB, ESP block was associated with less analgesic consumption, lower VAS pain scores, and a greater analgesic effect. Furthermore, the ESP block may offer considerable advantages over the TPVB in terms of ease of performance and safety, though this needs to be assessed in future studies.

\section{Abbreviations}

\section{TPVB: thoracic paravertebral block; ESP: erector spinae plane; BMI: Body mass index; ASA: American Society of Anesthesiologists; PCA: Patient-controlled analgesia; VAS: Visual analogue scale}

\section{Declarations}

Ethics approval and consent to participate

The study was approved by the ethics committee of Beijing Chaoyang Hospital (2017-ke-81). The trial was registered retrospectively at the Clinical Trial Registry of China (ChiCTR-INR-17011499). Each patient provided a written informed consent.

Consent for publication

Not applicable.

Availability of data and materials

The main data have been presented in the article and its additional file (Additional file 1). All the other data and materials supporting the conclusions of this article are available from the corresponding author on reasonable request.

Competing interests 
The authors declare that they have no competing interests.

Funding

This project was supported by grants from the National Natural Science Foundation of China(8160050435 to Danxu Ma) and the Fostering Program for High-level Health and Technical Personnel in Beijing (2013-3-018 to Yun Wang). The funding source had no role in the design of this study, analyses, interpretation of the data or writing the manuscript.

\section{Authors' Contributions}

DXM and HLR contributed equally to this work, in performing most of the experiments and writing the first draft manuscript. XYL, HLL, JJ and ASW performed or assisted with portions of the experiments and data analysis. YW designed and directed the project and wrote the final manuscript. All authors have read and approved the final manuscript.

Acknowledgments

The authors thank Hui Li and Bin Hu, from the Department of Chest Surgery, Beijing Chaoyang Hospital, Capital Medical University, Beijing, China, for their assistance in patient recruitment.

Authors' Information

${ }^{1}$ Department of Anesthesiology, Beijing Chaoyang Hospital, Capital Medical University, No. 8, Gongtinan Road, Chaoyang District, 100020, Beijing, China. ${ }^{2}$ Department of Anesthesiology, Dongzhimen Hospital, Beijing University of Chinese Medicine, No. 5 Haiyuncang, Dongcheng District, 100700, Beijing, China.

\section{References}

1.De Cosmo G, Aceto P, Gualtieri E, Congedo E. Analgesia in thoracic surgery: review. Minerva Anestesiol. $2009 ; 75: 393-400$.

2.Taylor R, Massey S, Stuart-Smith K. Postoperative analgesia in video-assisted thoracoscopy: the role of intercostal blockade. J Cardiothorac Vasc Anesth. 2004; 18(3):317-21.

3.Richardson J, Sabanathan S, Shah R. Post-thoracotomy spirometric lung function: the effect of analgesia. A review. J Cardiovasc Surg (Torino). 1999; 40:445-56.

4.Romero A, Garcia JE, Joshi GP. The state of the art in preventing postthoracotomy pain. Semin Thorac Cardiovasc Surg. 2013; 25:116-24.

5.Freise H, Van Aken HK. Risks and benefits of thoracic epidural anaesthesia. Br J Anaesth. 2011; 107:859-68. 
6.Bouman EAC, Sieben JM, Balthasar AJR, Joosten EA, Gramke HF, van Kleef M, Lataster A. Boundaries of the thoracic paravertebral space: potential risks and benefits of the thoracic paravertebral block from an anatomical perspective. Surg Radiol Anat. 2017; 39:1117-25.

7.Forero M, Adhikary SD, Lopez H, Tsui C, Chin KJ. The Erector Spinae Plane Block: A Novel Analgesic Technique in Thoracic Neuropathic Pain. Reg Anesth Pain Med. 2016; 41:621-7.

8.Hamilton DL, Manickam B: The Erector Spinae Plane Block. Reg Anesth Pain Med. 2017; 42:276.

9.Munoz F, Cubillos J, Bonilla AJ, Chin KJ. Erector spinae plane block for postoperative analgesia in pediatric oncological thoracic surgery. Can J Anaesth. 2017; 64:880-2.

10.Ueshima $\mathrm{H}$, Otake $\mathrm{H}$. Erector spinae plane block provides effective pain management during pneumothorax surgery. J Clin Anesth. 2017; 40:74.

11.Chin KJ, Malhas L, Perlas A. The Erector Spinae Plane Block Provides Visceral Abdominal Analgesia in Bariatric Surgery: A Report of 3 Cases. Reg Anesth Pain Med. 2017; 42:372-6.

12.Elkoundi A, Eloukkal Z, Bensghir M, Belyamani L, Lalaoui SJ. Erector Spinae Plane Block for Hyperalgesic Acute Pancreatitis. Pain Med. 2018.

13.Tulgar S, Kapakli MS, Senturk O, Selvi O, Serifsoy TE, Ozer Z. Evaluation of ultrasound-guided erector spinae plane block for postoperative analgesia in laparoscopic cholecystectomy: A prospective, randomized, controlled clinical trial. J Clin Anesth. 2018; 49:101-6.

14.Garutti I, Olmedilla L, Perez-Pena JM, Arnal D, Pineiro P, Barrigon S, Navia J. Hemodynamic effects of lidocaine in the thoracic paravertebral space during one-lung ventilation for thoracic surgery. $J$ Cardiothorac Vasc Anesth. 2006; 20:648-51.

15.Kasimahanti R, Arora S, Bhatia N, Singh G. Ultrasound-guided single-vs double-level thoracic paravertebral block for postoperative analgesia in total mastectomy with axillary clearance. J Clin Anesth. 2016; 33:414-21.

16.Ivanusic J, Konishi Y, Barrington MJ. A Cadaveric Study Investigating the Mechanism of Action of Erector Spinae Blockade. Reg Anesth Pain Med. 2018; 43:567-71.

17.Adhikary SD, Bernard S, Lopez H, Chin KJ. Erector Spinae Plane Block Versus Retrolaminar Block: A Magnetic Resonance Imaging and Anatomical Study. Reg Anesth Pain Med. 2018; 43:756-62.

18.Greenhalgh K, Womack J, Marcangelo S. Injectate spread in erector spinae plane block. Anaesthesia. $2019 ; 74: 126-7$.

19.Elsharkawy H, Pawa A, Mariano ER. Interfascial Plane Blocks: Back to Basics. Reg Anesth Pain Med. 2018; 43:341-6. 
20.Schoenfeldt J, Guffey R, Fingerman M. Cadaveric study investigating the mechanism of action of erector spinae blockade. Reg Anesth Pain Med. 2019.

21.El-Boghdadly K, Pawa A. The erector spinae plane block: plane and simple. Anaesthesia. 2017; 72:434-8.

\section{Tables}

Table 1 General characteristics of the patients

\begin{tabular}{|c|c|c|c|c|}
\hline Characteristic & $\begin{array}{c}\text { Control group }(\mathrm{n}= \\
\text { 19) }\end{array}$ & $\underset{20)}{\operatorname{TPVB} \text { group }}(\mathrm{n}=$ & ESP group $(n=$ & $\mathrm{P}$ values \\
\hline Age (y) & $53.4(8.5)$ & $52.9(7.6)$ & $54.1(9.4)$ & NS \\
\hline Sex & $10(53 \%)$ & $12(60 \%)$ & $9(43 \%)$ & NS \\
\hline Male & $9(47 \%)$ & $8(40 \%)$ & $12(57 \%)$ & \\
\hline $\begin{array}{l}\text { Body mass index }\left(\mathrm{kg} / \mathrm{m}^{2}\right) \\
\text { ASA status }\end{array}$ & $24.5(4.7)$ & $26.8(6.5)$ & $23.6(5.8)$ & NS \\
\hline I & $\begin{array}{l}7(37 \%) \\
12(63 \%)\end{array}$ & $\begin{array}{l}8(40 \%) \\
12(60 \%)\end{array}$ & $\begin{array}{l}11(52 \%) \\
10(48 \%)\end{array}$ & $\begin{array}{l}\text { NS } \\
\text { NS }\end{array}$ \\
\hline $\begin{array}{l}\text { Duration of surgery } \\
\text { (min) }\end{array}$ & $179.3(27.6)$ & 190.5 (30.7) & $187.2(32.6)$ & NS \\
\hline
\end{tabular}

Values are presented as mean \pm SD or number of patients (\%)

Abbreviations: TPVB thoracic paravertebral block, ESP erector spinae plane, ASA American society of anesthesiologists, NS Not significant

Table 2 Hemodynamic fluctuations after the administration of regional anesthesia

\begin{tabular}{|c|c|c|c|c|}
\hline Parameter & $\begin{array}{c}\text { Control group } \\
(\mathrm{n}=19)\end{array}$ & $\begin{array}{c}\text { TPVB group } \\
(\mathrm{n}=20)\end{array}$ & $\begin{array}{l}\text { ESP group } \\
(\mathrm{n}=21)\end{array}$ & P values \\
\hline \multicolumn{5}{|c|}{ Mean arterial pressure (mmHg) } \\
\hline Before block & $90.2 \pm 8.1$ & $89.2 \pm 9.1$ & $90.6 \pm 7.3$ & NS \\
\hline 5 min after block & $89.3 \pm 8.7$ & $87.3 \pm 8.8$ & $89.7 \pm 7.7$ & \\
\hline 15 min after block & $88.6=$ & 86.6 & 8.1 & \\
\hline 30 min after block & $89.6 \pm 7.9$ & $85.6 \pm 7.2$ & $86.1 \pm 7.8$ & $\mathbf{N}$ \\
\hline \multicolumn{5}{|l|}{ Heart rate $(\mathrm{bpm})$} \\
\hline Before block & $78.2 \pm 7.8$ & $75.6 \pm 8.3$ & $77.6 \pm 7.7$ & NS \\
\hline 5 min after block & $77.9 \pm 6.9$ & $77.3 \pm 7.9$ & $78.4 \pm 8.3$ & NS \\
\hline 15 min after block & $77.4 \pm$ & $76.4 \pm 9.6$ & $79.6 \pm 7.2$ & NS \\
\hline 30 min after block & $78.8 \pm 7.4$ & $72.8 \pm 8.4$ & $75.1 \pm 9.4$ & NS \\
\hline
\end{tabular}

Values are presented as mean $\pm \mathrm{SD}$

Abbreviations: TPVB thoracic paravertebral block, ESP erector spinae plane, ASA American society of anesthesiologists, NS Not significant

Table 3 Postoperative complications

\begin{tabular}{lcccc}
\hline Adverse effect & $\begin{array}{c}\text { Control group } \\
(\mathrm{n}=19)\end{array}$ & TPVB group $(\mathrm{n}=20)$ & ESP group $(\mathrm{n}=21)$ & $P$ value \\
\hline Nausea & $13(68.4 \%)$ & $12(60 \%)$ & $14(66.7 \%)$ & NS \\
Vomiting & $4(21.1 \%)$ & $3(15 \%)$ & $3(14.3 \%)$ & NS \\
\hline
\end{tabular}

Data are given as number of patients (\%).

Abbreviations: TPVB thoracic paravertebral block, ESP erector spinae plane

\section{Figures}




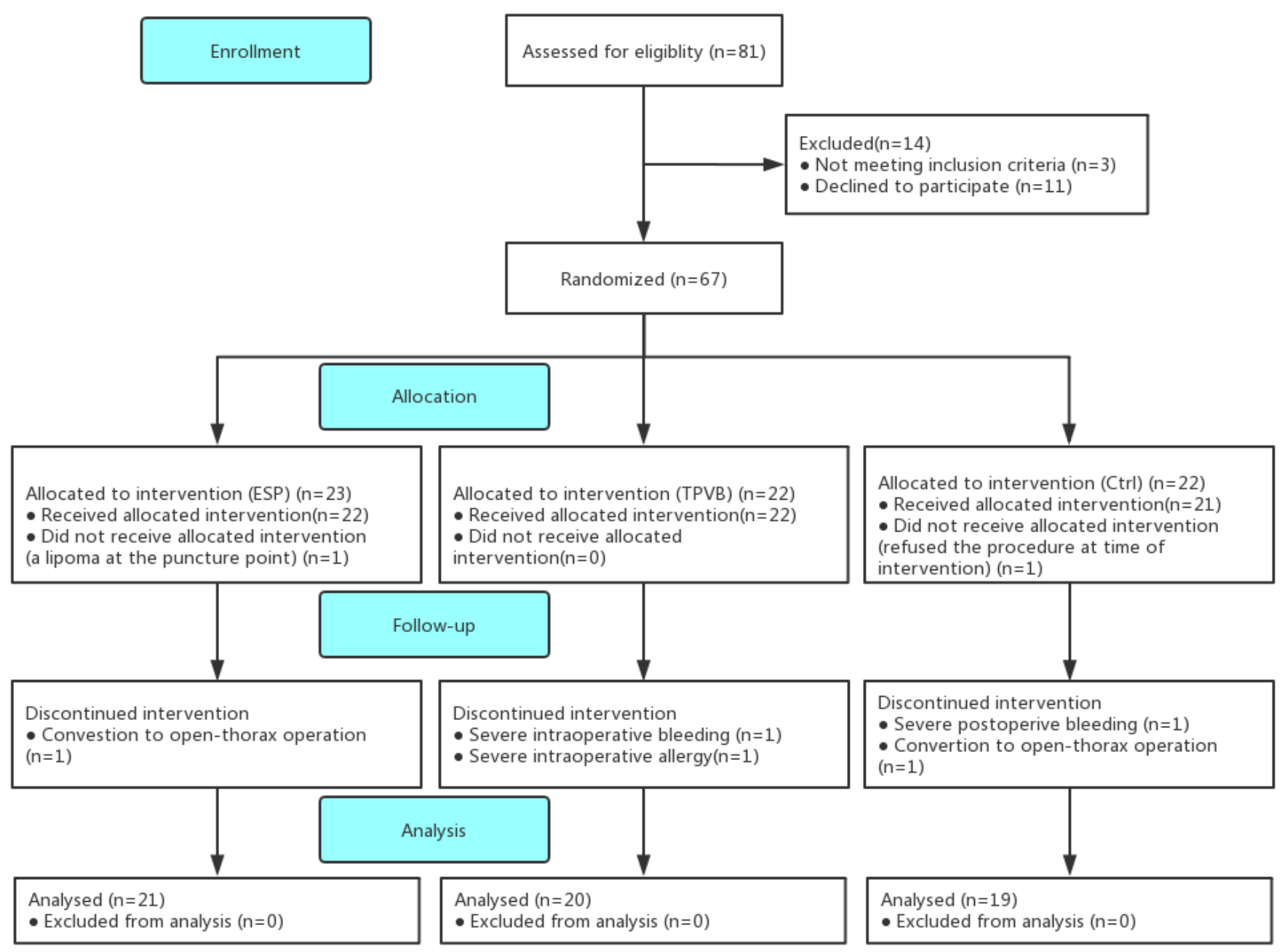

Figure 1

Flowchart of subject enrollment, allocation, randomization, follow-up and analysis. 


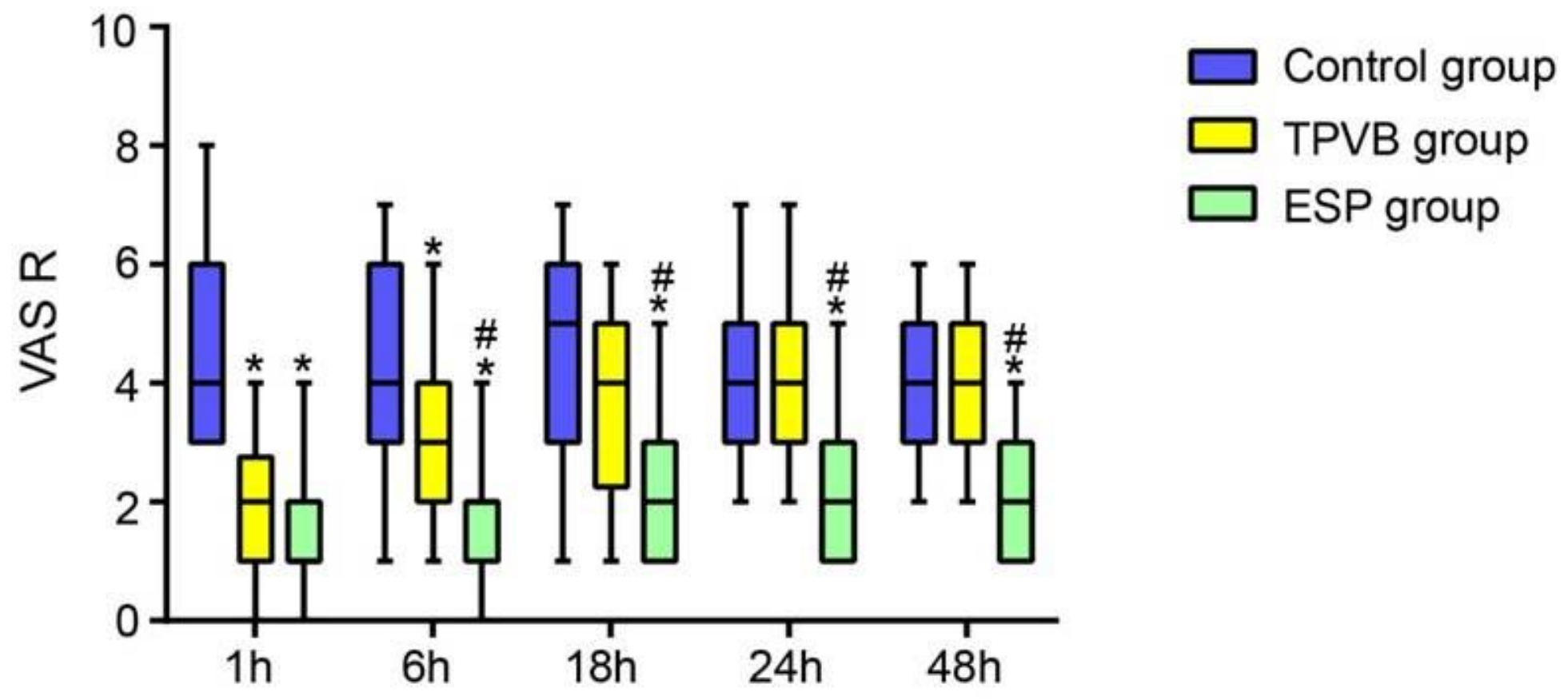

Figure 2

VAS at rest at 1, 6, 18, 24 and 48 hours after operation. The boxes represent interquartile range. The whiskers represent the lower and upper adjacent values. The horizontal line within the boxes represents median. *P $<0.05$ compared with the control group; \#P $<0.05$ compared with the TPVB group.

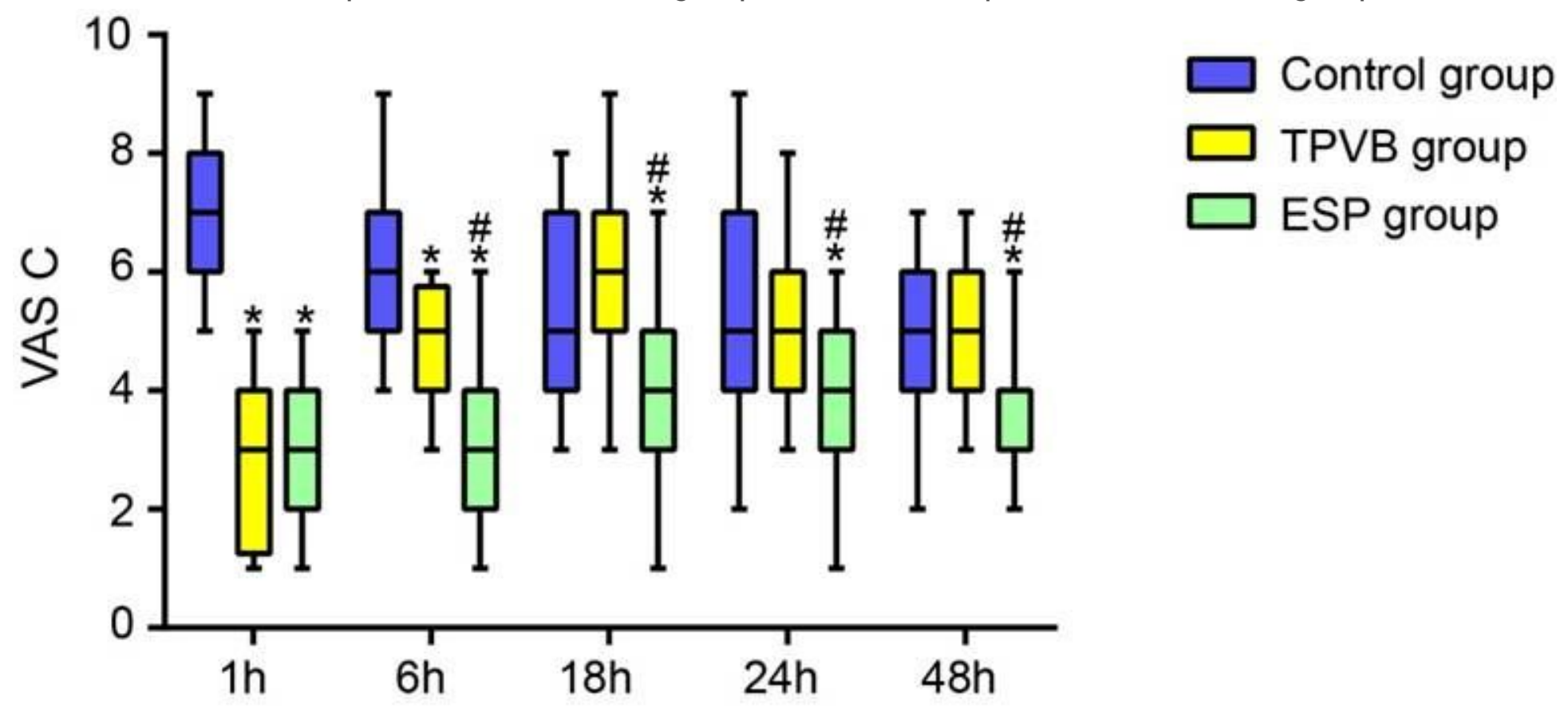

Figure 3

VAS at coughing at 1, 6, 18, 24 and 48 hours after operation. The boxes represent interquartile range. The whiskers represent the lower and upper adjacent values. The horizontal line within the boxes represents median. ${ }^{*} \mathrm{P}<0.05$ compared with the control group; \#P $<0.05$ compared with the TPVB group. 

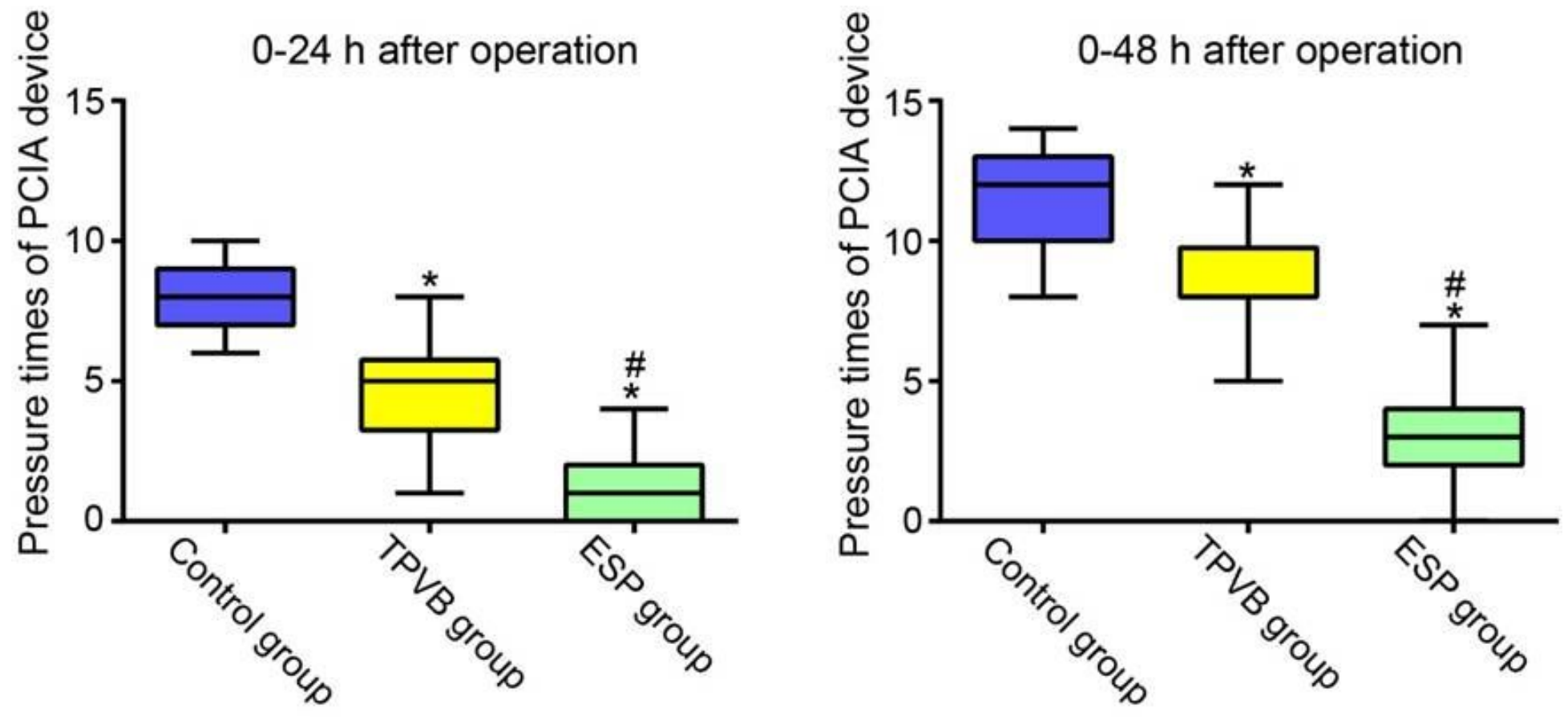

Figure 4

The number of PCIA doses delivered. The boxes represent interquartile range. The whiskers represent the lower and upper adjacent values. The horizontal line within the boxes represents median. ${ }^{*} \mathrm{P}<0.05$ compared with the control group; \#P $<0.05$ compared with the TPVB group.

\section{0-24 $\mathrm{h}$ after operation}

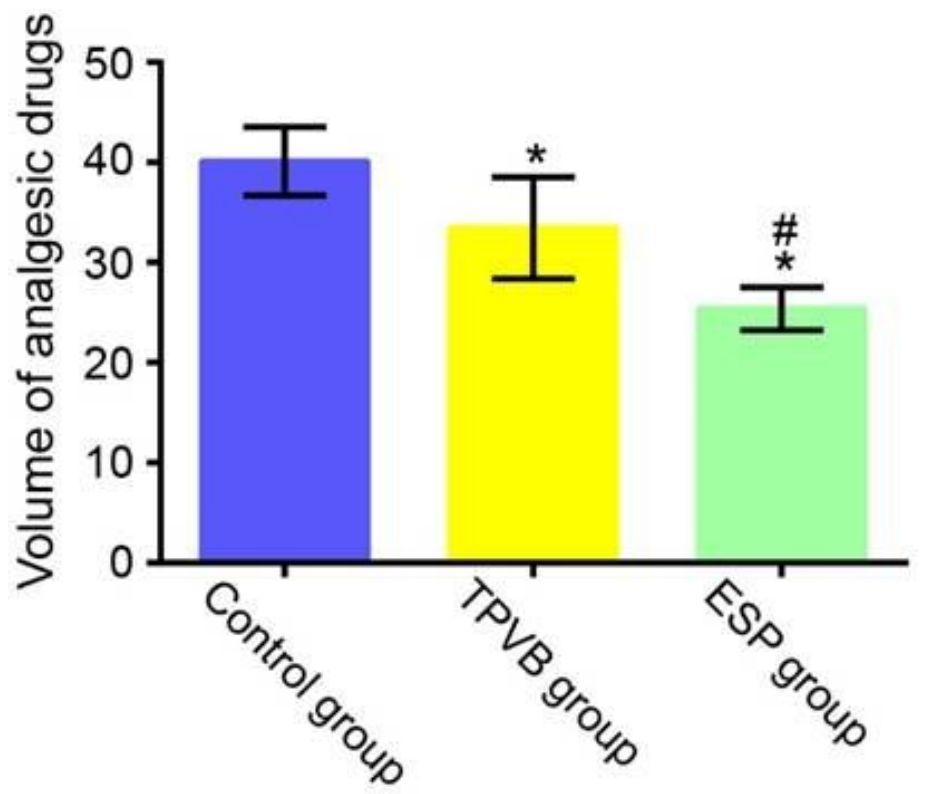

0-48 h after operation

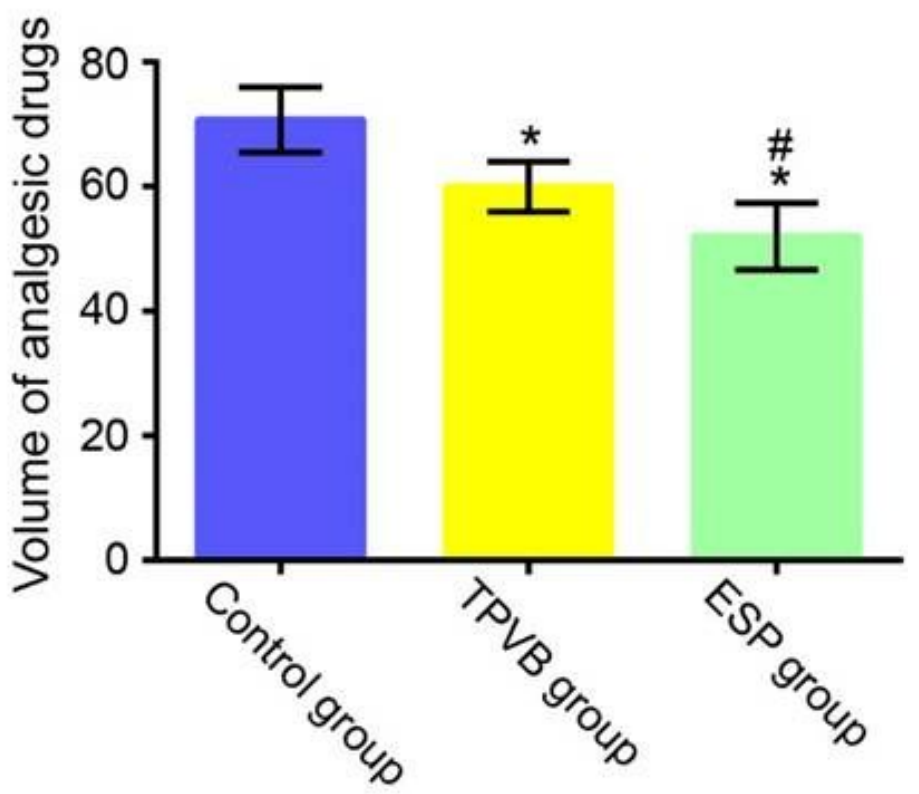

\section{Figure 5}

The volume of analgesic drugs administered via the PCIA device. ${ }^{*} \mathrm{P}<0.05$ compared with the control group; \#P $<0.05$ compared with the TPVB group. 
0-24 $\mathrm{h}$ after operation

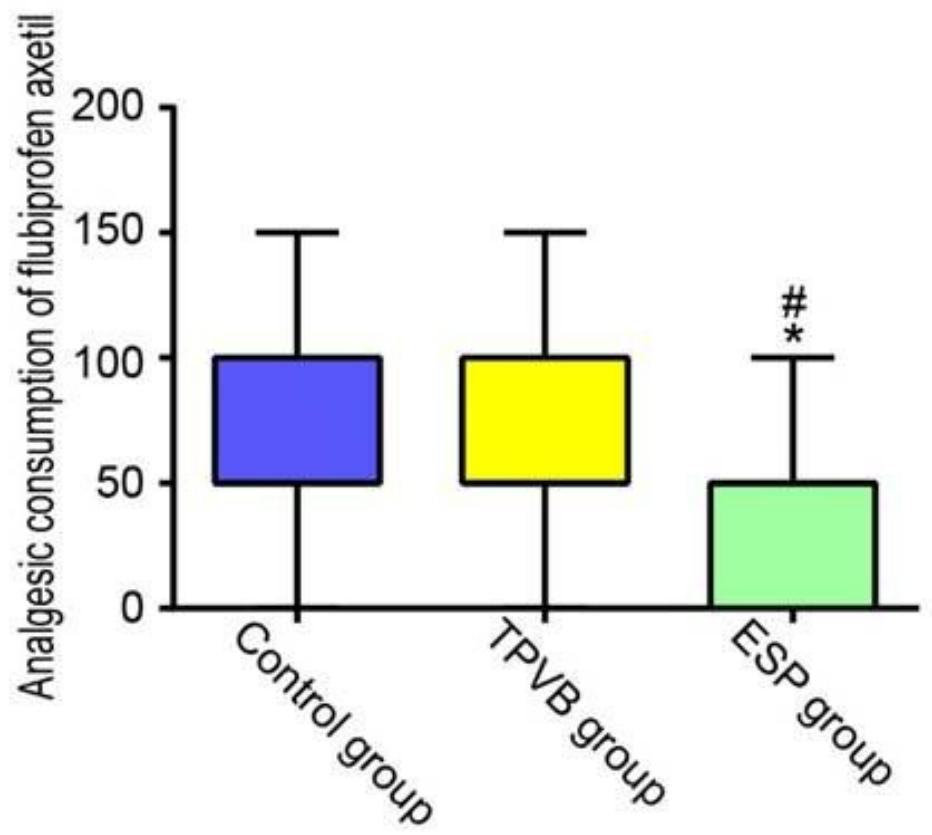

$0-48 \mathrm{~h}$ after operation

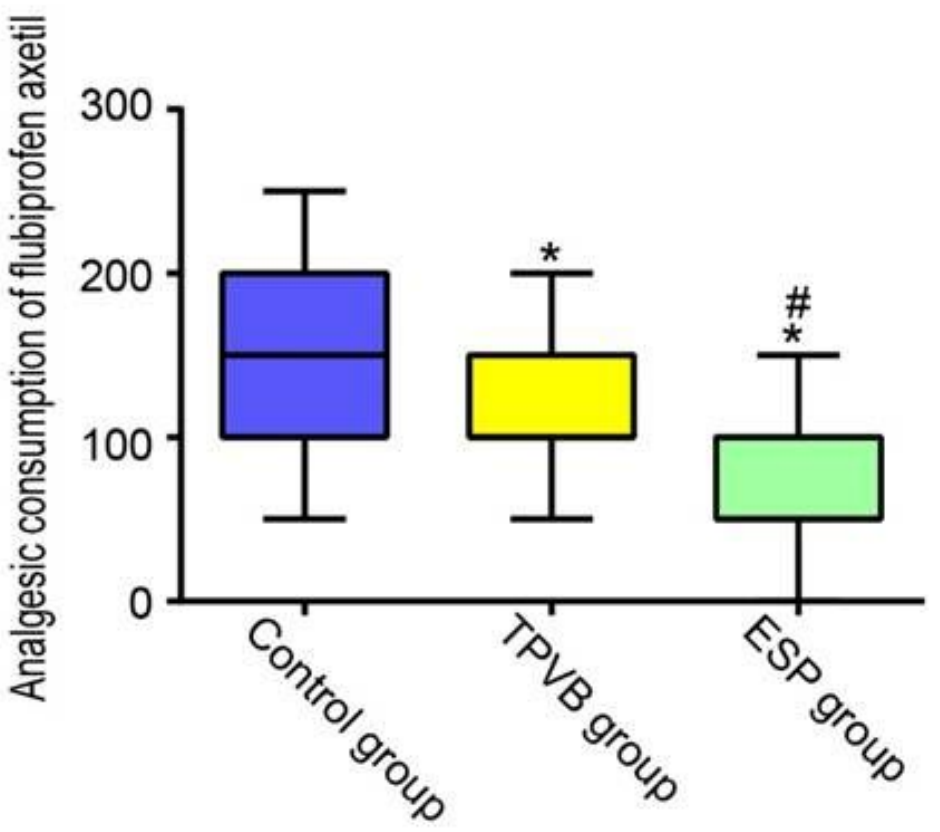

\section{Figure 6}

The consumption of flurbiprofen axetil. The boxes represent interquartile range. The whiskers represent the lower and upper adjacent values. The horizontal line within the boxes represents median. ${ }^{*} \mathrm{P}<0.05$ compared with the control group; \#P $<0.05$ compared with the TPVB group.

\section{Supplementary Files}

This is a list of supplementary files associated with this preprint. Click to download.

- Additionalfile1.xlsx 